\title{
Submillimeter-Wave 3.3-bit RF MEMS Phase Shifter Integrated in Micromachined Waveguide
}

\author{
Umer Shah, Member, IEEE, Emmanuel Decrossas, Member, IEEE, Cecile Jung-Kubiak, Member, IEEE, \\ Theodore Reck, Member, IEEE, Goutam Chattopadhyay, Fellow, IEEE, Imran Mehdi, Fellow, IEEE, \\ and Joachim Oberhammer, Senior Member, IEEE
}

\begin{abstract}
This paper presents a submillimeter-wave 500$550 \mathrm{GHz}$ MEMS-reconfigurable phase shifter which is based on loading a micromachined rectangular waveguide with 9 E-plane stubs. The phase shifter uses MEMS-reconfigurable surfaces to individually block/unblock the E-plane stubs from the micromachined waveguide. Each MEMS-reconfigurable surface is designed so that in the non-blocking state it allows the electromagnetic wave to pass freely through it into the stub while in the blocking state it serves as the roof of the main waveguide and blocks the wave propagation into the stub. The phase-shifter design comprises three micromachined chips that are mounted in the $\mathrm{H}$-plane cuts of the rectangular waveguide. Experimental results of the first device prototypes show that the MEMS-reconfigurable phase shifter has a linear phase shift of $20^{\circ}$ in ten discrete steps $(3.3 \mathrm{bit})$. The measured insertion loss is better than $3 \mathrm{~dB}$, of which only 0.5 to $1.5 \mathrm{~dB}$ is attributed to the MEMS-surfaces and switched stubs, and the measured return loss is better than $15 \mathrm{~dB}$ in the design frequency band of 500$550 \mathrm{GHz}$. It is also shown that the major part of the insertion loss is attributed to misalignment and assembly uncertainties of the micromachined chips and the waveguide flanges, shown by simulations and reproducibility measurements. The MEMSreconfigurable phase shifter is also operated in an analog tuning mode for high phase resolution. Furthermore, a detailed study has been carried out identifying the reason for the discrepancy between the simulated $\left(90^{\circ}\right)$ and the measured $\left(20^{\circ}\right)$ phase shift. Comb-drive actuators with spring constant variations between 2.13 to $8.71 \mathrm{~N} / \mathrm{m}$ are used in the phase shifter design. An actuation voltage of $21.94 \mathrm{~V}$ with a reproducibility better than $\sigma=0.0503 \mathrm{~V}$ is measured for the actuator design with a spring constant of $2.13 \mathrm{~N} / \mathrm{m}$. Reliability measurement on this actuator was performed in an uncontrolled laboratory environment and showed no deterioration in the functioning of the actuator observed over one hundred million cycles.
\end{abstract}

Index Terms-RF MEMS, phase shifter, micromachined waveguide, submillimeter-wave, rectangular waveguide, terahertz.

\section{INTRODUCTION}

$\mathbf{T}$ $\mathrm{HE}$ terahertz frequency band is one of the least utilized regions of the electromagnetic spectrum despite of all the scientific and industrial interest and potential [1], largely due to

Umer Shah and Joachim Oberhammer are with the Micro and Nanosystems, School of Electrical Engineering, KTH Royal Institute of Technology, Stockholm SE-100 44, Sweden (e-mail: umers@kth.se; joachim.oberhammer@ee.kth.se).

Emmanuel Decrossas, Cecile Jung-Kubiak, Theodore Reck, Goutam Chattopadhyay and Imran Mehdi are with the Jet Propulsion Laboratory, California Institute of Technology, Pasadena, CA 91109 USA (e-mail: Theodore.reck@jpl.nasa.gov).

Manuscript received XXXXX; revised XXXXX. the unavailability of commercial off-the-shelf submillimeterwave components [2] and the lack of effective methods to characterize the circuits [3]. Terahertz or the submillimeterwave frequency band has emerging applications in radar, radio astronomy, imaging systems, medical diagnosis, spectral analysis and high bandwidth wireless communications [4]. Waveguides are used as a typical transmission medium for submillimeter-wave circuits as they exhibit very low insertion loss when compared to planar transmission lines at these frequencies. Rectangular waveguides can be fabricated by utilizing metal machining techniques achieving a typical tolerance of approximately $5 \mu \mathrm{m}$ [5] with many restrictions in geometries. For waveguide based designs in the WR-1.5 band, the fabrication accuracy should be at least $5 \mu \mathrm{m}$ [5]. This fabrication accuracy requirement becomes even more stringent when dealing with waveguide resonant structures [5]. In order to improve the accuracy and the precision of the fabricated dimensions, silicon micromachining techniques have emerged as a very attractive alternative [6]. Furthermore, silicon micromachining has the advantages of high-volume, low-cost batch fabrication, high product uniformity and with the smallest dimensions determined through photolithography. Waveguides fabricated utilizing silicon micromachining techniques have already shown promising results for frequencies as high as $2.7 \mathrm{THz}$ [7].

A rectangular waveguide loaded with stub sections can be used for building low insertion loss static phase shifters [8], waveguide tuners [9], leaky wave antennas [10] and filters [11]. Variable phase shifters are widely employed in phased-array antennas for beam steering, used in radar and communication applications. Conventional electronic phase shifters based on p-i-n diode switches, MESFET, and GaAs usually have high insertion loss, large power consumption, and poor linearity, in particular at sub-THz frequencies [12]. Ferrite based phase shifters show better performance, but are not commercially viable because of their large size and high cost and would be prohibitively loss at terahertz frequencies. Recently, phase shifters utilizing liquid crystals have been introduced [13] but they suffer from slow response time, usually in seconds. Microelectromechanical systems (MEMS) based phase shifters have high linearity and low insertion loss over a large bandwidth when compared to their solid-state counterparts [12] and have shown promising performance up to $110 \mathrm{GHz}$ frequency [12]. In all literature, the maximum operational frequency that MEMS-based devices have been reported at is $220 \mathrm{GHz}$ [14], and these designs were imple- 
mented on planar transmission lines resulting in high losses at submillimeter-wave frequencies.

The unavailability of reconfigurable waveguide components at submillimeter-wave frequencies provides an excellent and so far unexploited opportunity for tunable MEMS components. This paper introduces the first ever submillimeter-wave MEMS reconfigurable phase shifter operating in the frequency band between 500 to $550 \mathrm{GHz}$. In two recent conference publications, the authors have shown how a MEMS switched surface can be used to make an RF MEMS switchable stub [15] and how to use such switchable stubs for phase shifting [16]. This paper is an extension of these conference papers [15], [16], and discusses the phase shifter design, fabrication and assembly in detail, and new measurement data (both RF and electromechanical) is presented. A detailed analysis of the discrepancy between simulated and measured results is carried out. To the best knowledge of the authors, the phase shifter reported in this paper is the first ever MEMS reconfigurable component at submillimeter-wave frequency. It is the first reported RF MEMS component to be operating above $220 \mathrm{GHz}$. Additionally, it is the first MEMS waveguide component and the first MEMS phase shifter operating above $110 \mathrm{GHz}$.

\section{CONCEPT AND DESIGN}

The phase shifter concept is shown in Fig. 1. It is based on loading a rectangular waveguide with E-plane waveguide stubs [8]. The E-plane stubs are connected to the H-plane top roof of the rectangular waveguide. This results in a series reactance which is tuned by making the E-plane stubs switchable by using MEMS-reconfigurable E-plane surfaces [17]. The MEMS-reconfigurable surfaces are inserted into the top roof of the rectangular waveguide perpendicular to the electromagnetic wave propagation into the waveguide stubs.

The MEMS-reconfigurable E-plane surface consist of a set of fixed vertical cantilevers, shown in yellow in Fig. 1(b), that are anchored and a set of synchronously movable vertical cantilevers, shown in brown in Fig. 1(b), that are mechanically connected to electrostatic comb-drive MEMS actuators via horizontal suspension beams. The movable vertical cantilevers can be displaced in the horizontal direction so that they either block or unblock the $\mathrm{TE}_{10}$ wave propagation mode into the waveguide stubs. In the non-blocking (open) state of the MEMS-reconfigurable surface, there is a gap between the fixed and the movable vertical cantilevers which allows the electromagnetic wave to propagate freely through the reconfigurable surface making it transmissive in this state. In the blocking (closed) state of the MEMS-reconfigurable surface, the movable vertical cantilevers are laterally displaced by MEMS actuators and brought into contact with the nonmovable vertical cantilevers. This forms a series of closed columns of vertical cantilevers, blocking the electromagnetic wave propagation through the switched surface by shortcircuiting the electric field lines of the $\mathrm{TE}_{10}$ mode making it reflective in this state. In the blocking state, the MEMSswitched surface also carries the roof currents and forms the roof of the rectangular waveguide. Thus, the surface has a different electrical function in the two states, with different

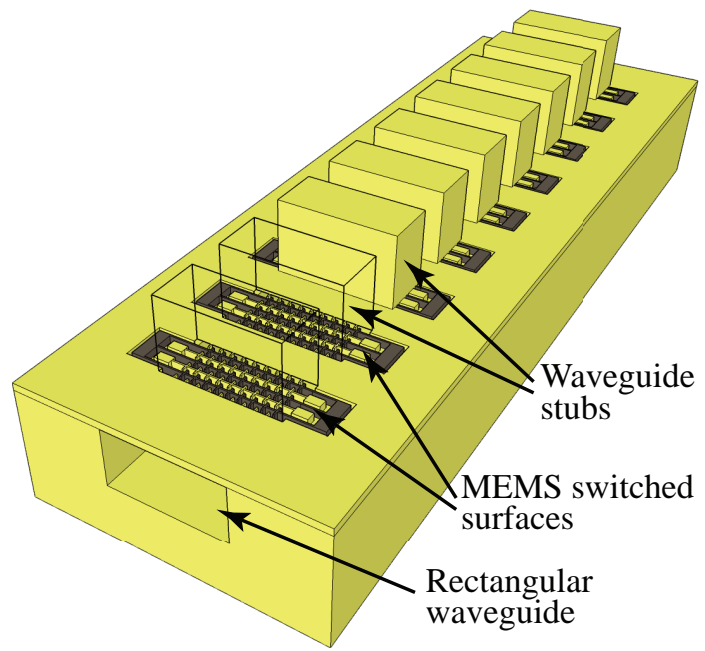

(a)

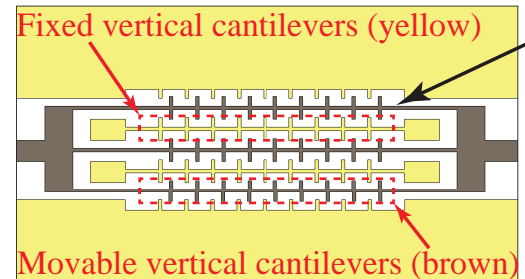

Open surface (non blocking)
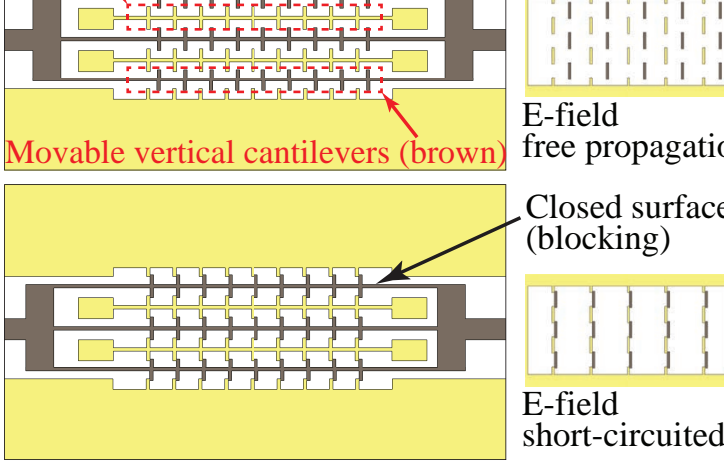

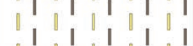

E-field

free propagation

(b)

Fig. 1. Submillimeter-wave MEMS phase shifter design based on a micromachined rectangular waveguide loaded with 9 E-plane stubs. The stubs are switched by using MEMS reconfigurable surfaces: (a) 3-D illustration; and (b) Non-blocking and blocking state of the MEMS-reconfigurable surface.

requirements on its implementation. The number of vertical cantilevers and horizontal bars have a strong influence on the ability of the MEMS-switched surface to block or unblock the wave propagation into the stub [17]. This number is optimized for a compromise between a low loss transmission into the stub in the non blocking state and a high isolation with a good configuration for the roof-currents in the blocking state.

Fig. 2 shows the E-field of a single switchable stub loading a rectangular waveguide at $550 \mathrm{GHz}$ simulated using CST Microwave Studio, for the nonblocking state [Fig. 2(a)] and the blocking state [Fig. 2(b)]. The implemented rectangular waveguide is loaded with 9 such stubs each with a length of $300 \mu \mathrm{m}$ and a nominal phase shift of $10^{\circ}$ producing a total phase shift of $90^{\circ}$ in $10^{\circ}$ steps (3.3 bit). Fig. 3 shows the simulated phase shift for different vertical cantilever gaps normalized to the state with all stubs unblocked. The design parameter study to select the nominal gap of the vertical cantilevers in the non-blocking state shows only a minor improvement $(2 \%)$ in the phase shift when the gap in the nonblocking state is increased from $5 \mu \mathrm{m}$ to $10 \mu \mathrm{m}$. A $5 \mu \mathrm{m}$ initial 


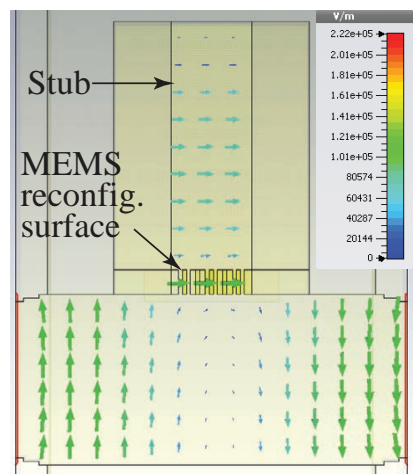

(a)

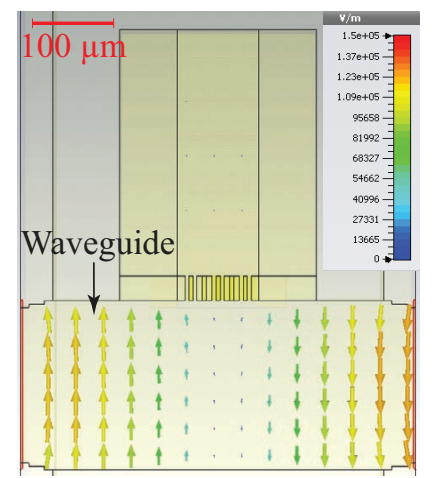

(b)
Fig. 2. Simulated electric field distribution in the E-plane stub loading a rectangular waveguide in the two actuation states of the the MEMS reconfigurable surface: (a) non-blocking state; and (b) blocking state.

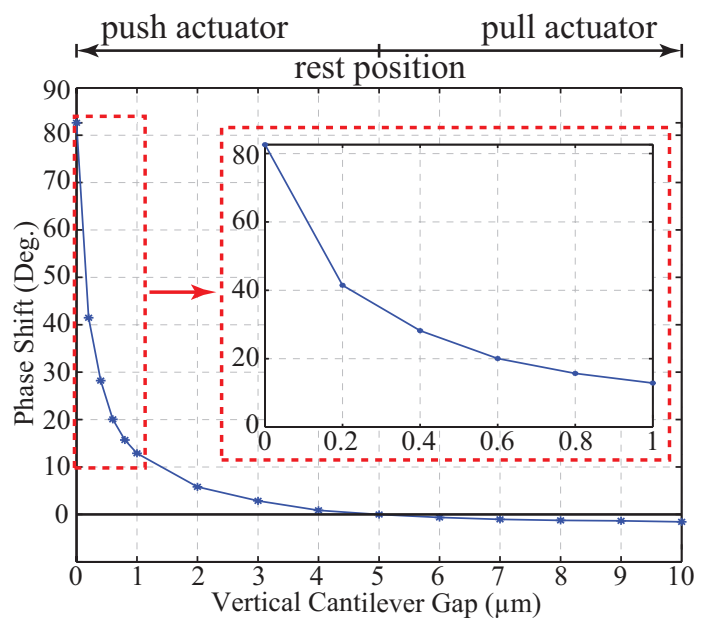

(a)

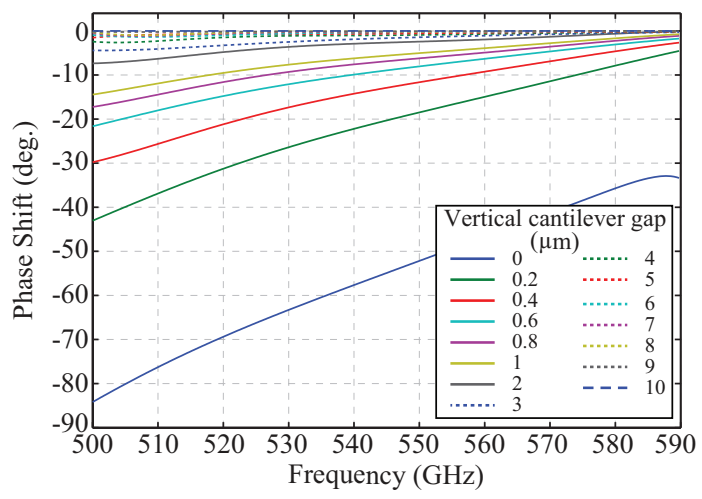

(b)

Fig. 3. Design parameter study of the waveguide phase shifter to evaluate the influence of the vertical cantilever gap: (a) simulated phase shift at $500 \mathrm{GHz}$ as a function of vertical cantilever gap; and (b) simulated phase shift for different vertical cantilever gaps as a function of frequency.

gap is selected in the non-blocking state for the design with an overlap of $2 \mu \mathrm{m}$ between the vertical cantilevers. Fig. 3(b) shows that the maximum phase shift is achieved when there is an ohmic contact between the vertical cantilevers in the blocking state. However, an ohmic contact may not be needed since a gap of $200 \mathrm{~nm}$ between the vertical cantilevers in the blocking state also results in a phase shift, even though reduced

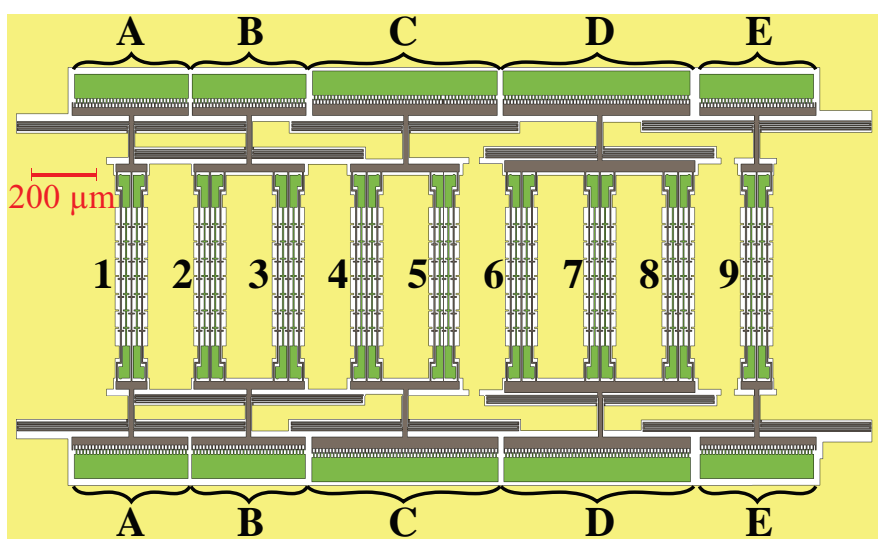

Fig. 4. Illustration of the MEMS switched stub chip with close-up of the MEMS switched surfaces and the comb-drive actuators.

TABLE I

Relation Between the Actuators, Stub Numbers and Stub GROUPS

\begin{tabular}{ccc}
\hline Stub Group & Stub Number & Connected Actuator \\
\hline A & 1 & Actuator A \\
B & 2,3 & Actuator B \\
C & 4,5 & Actuator $\mathbf{C}$ \\
D & $6,7,8$ & Actuator $\mathbf{D}$ \\
E & 9 & Actuator $\mathbf{E}$ \\
\hline
\end{tabular}

by half.

The rectangular waveguide used for the phase shifter design has non-standard dimensions $(0.416 \mathrm{~mm} \times 0.208 \mathrm{~mm})$ optimized for the lower WR-1.5 band. The phase shifter is composed of three vertically stacked chips, a MEMS chip with the MEMS-switched surfaces and the stubs, a micromachinedwaveguide chip, and a cap chip terminating the stubs. Electrostatic comb-drive MEMS actuators with folded-beam suspensions are symmetrically placed around the waveguide stubs on the MEMS-switched stub chip with five comb-drive actuators on each side to move the vertical cantilevers of the MEMSswitched surfaces for the 9 stubs. The total displacement of the vertical cantilevers is $10 \mu \mathrm{m}$ or $\pm 5 \mu \mathrm{m}$ around the rest position using a push-pull actuator. Fig. 4 shows the layout of the MEMS switched stub chip. The number of actuators in the phase shifter design is optimized to achieve all the ten phase states with the minimum number of actuated combdrives. Table I shows the grouping of the 9 stubs to the 5 MEMS actuators. Table II shows which actuator combination needs to be activated to achieve a certain phase state. As can be seen from Table II, the stubs are also grouped in a way to achieve maximum redundancy i.e., certain phase shifts can be achieved in multiple ways. For instance, a $40^{\circ}$ phase shift can be achieved either by actuating the combinations $[\mathrm{A}, \mathrm{B}, \mathrm{E}]$ or [D, E].

The layout of the MEMS reconfigurable surface is shown in Fig. 5(a). Two vertical cantilever widths of 4 and $5 \mu \mathrm{m}$ are used in the design. The different widths of the vertical cantilevers do not affect the measurement results and are only intended 
TABLE II

Actuator Combinations Used to Achieve the Designed Nominal Phase Shifts. Multiple Combinations Possible to Achieve $10^{\circ}$, $40^{\circ}, 60^{\circ}, 70^{\circ}$ AND $80^{\circ}$.

\begin{tabular}{|c|c|c|c|c|c|c|c|c|c|c|c|c|c|c|c|}
\hline \multicolumn{16}{|c|}{ Phase Shift (Deg.) } \\
\hline & 0 & & & 20 & 30 & 4 & & 50 & & & & & & & 90 \\
\hline Actuator A & 0 & 1 & 0 & 1 & 1 & 0 & 1 & 1 & 0 & 1 & 1 & 1 & 0 & 1 & 1 \\
\hline Actuator B & 0 & 0 & 0 & 0 & 1 & 0 & 1 & 1 & 0 & 1 & 0 & 1 & 1 & 1 & 1 \\
\hline Actuator C & 0 & 0 & 0 & 0 & 0 & 0 & 0 & 1 & 1 & 1 & 1 & 0 & 1 & 1 & 1 \\
\hline Actuator D & 0 & 0 & 0 & 0 & 0 & 1 & 0 & 0 & 1 & 0 & 1 & 1 & 1 & 1 & 1 \\
\hline Actuator E & 0 & 0 & 1 & 1 & 0 & 1 & 1 & 0 & 1 & 1 & 1 & 1 & 1 & 0 & 1 \\
\hline
\end{tabular}

$1=$ Actuated state, blocking the associated stubs; $0=$ Non actuated state, unblocking the associated stubs.

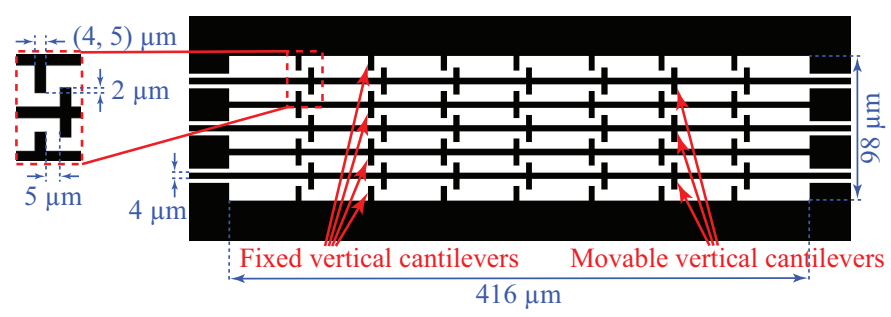

(a)

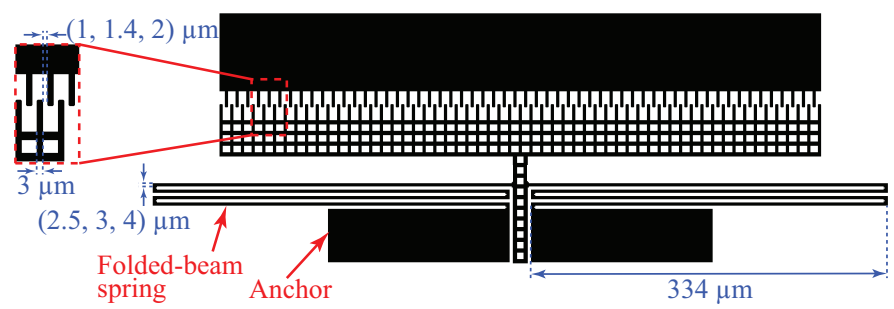

(b)

Fig. 5. Layout with nominal dimensions: (a) MEMS reconfigurable surface; and (b) electrostatic comb-drive MEMS actuator.

to overcome issues occurring due to the non-optimized fabrication process. Fig. 5(b) shows the layout of a designed single comb-drive actuator which is mirrored around the Eplane of the stub resulting in two comb-drive actuators for each stub group with a total of 10 actuators for 5 stub groups. The second actuator set is used to implement a push-pull configuration resulting in the device being actively opened and closed which allows for faster operation and larger travelling range. It should be noted that the phase shifter is designed for requiring two separate control signals for this push-pull operation. However, since a movement of $5 \mu \mathrm{m}$, already results in $98 \%$ of the total achievable phase shift [Fig. 3(a)], for all characterization in the paper, only a single-side actuator is employed. The number of fingers used for Actuator A, B and E is 34 , while 56 fingers are used for Actuator $\mathrm{C}$ and D. Different comb drives are designed with variations in spring width and finger gap resulting in different spring constants. The combdrive actuators do not require any dielectric insulation layers that are susceptible to dielectric charging and thus affect the actuation voltage repeatability and decrease reliability. Instead, mechanical stoppers are implemented for limiting the actuator movements. Monocrystalline silicon is used as the fabrication material for all the moving parts providing high structural

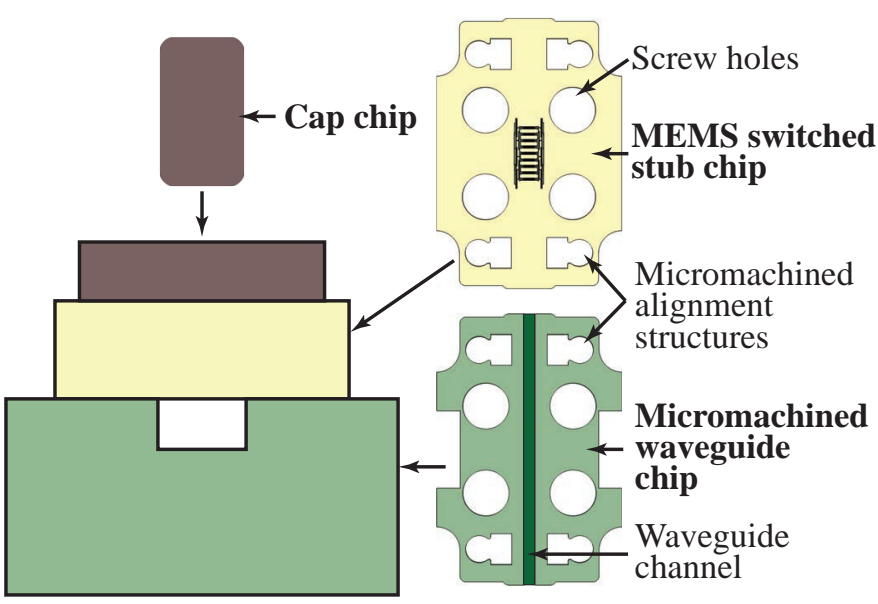

Fig. 6. Arrangement of the micromachined waveguide chip (bottom), MEMS switched stub chip (middle) and cap chip (top) to assemble the phase shifter.

reliability.

\section{FABRICATION AND ASSEMBLY}

The phase shifter comprises a MEMS-switched stub chip with the MEMS-switched surfaces and the stubs, a micromachined waveguide chip, and a cap chip terminating the stubs, as shown in Fig. 6. The MEMS-switched stub chip is fabricated in a two mask micromachining process outlined in Fig. 7 using a silicon-on-insulator (SOI) wafer. The fabrication begins with manufacturing the waveguide stubs by structuring the handle wafer using deep reactive ion etching (DRIE) [Fig. 7(b)]. This is followed by manufacturing the MEMS-reconfigurable surfaces by DRIE of the device layer [Fig. 7(c)]. Hydrofluoric acid (HF) is used for wet etching of the buried oxide (BOX) layer for free etching the MEMS moving structures which is followed by drying the MEMS chip in a supercritical drying step [Fig. 7(d)]. The chip is metallized by sputtering a $700 \mathrm{~nm}$ thick layer of gold on the handle wafer and a $200 \mathrm{~nm}$ thick layer of gold on the device layer using a $50 \mathrm{~nm}$ thick titanium tungsten adhesion layer [Fig. 7(e)]. Finally, a $50 \mathrm{~nm}$ layer of titanium tungsten as anti-stiction layer is sputtered on the device layer to complete the process. Fig. 8 shows a close-up SEM image of the fabricated MEMS switched stub chip. The micromachined waveguide chip is fabricated by structuring a silicon substrate using DRIE to make the waveguide channel. A $1 \mu \mathrm{m}$ thick gold layer is sputtered to metallize the chip. The 


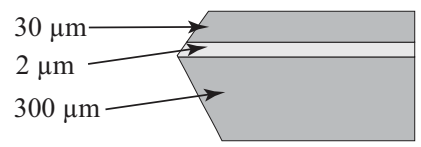

(a)

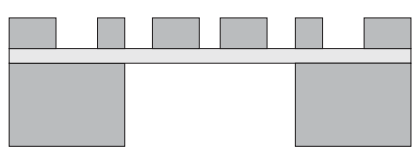

(c)

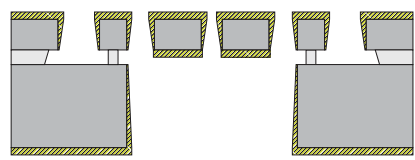

(e)

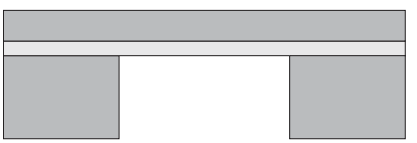

(b)

(d)

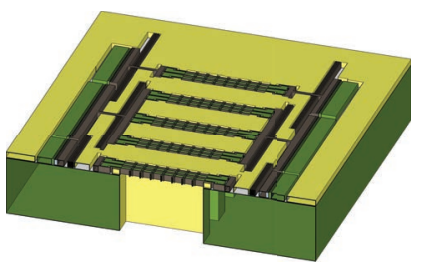

(f)

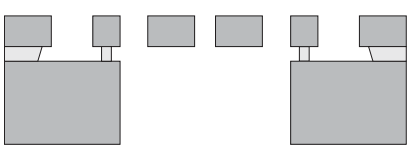

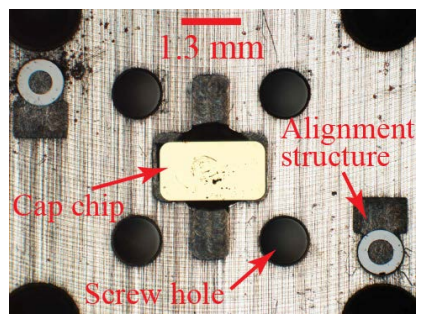

(a)

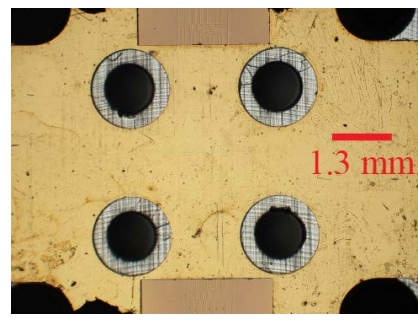

(c)

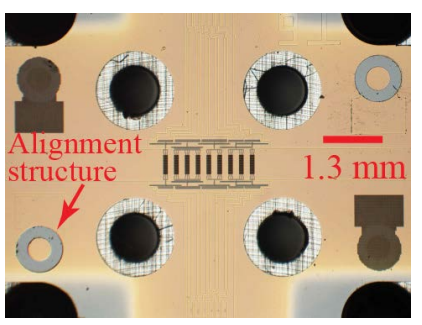

(b)

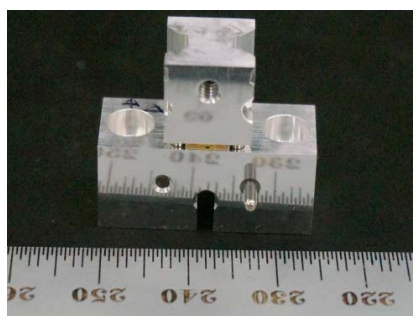

(d)
Fig. 7. Fabrication process of the MEMS-switched stub chip: (a) SOI wafer; (b) structuring of the handle wafer using DRIE; (c) structuring of the device layer using DRIE; (d) free etching using hydrofluoric acid; (e) metallization using sputtering; and (f) 3D illustration showing the cross-sectional view of the finished chip.

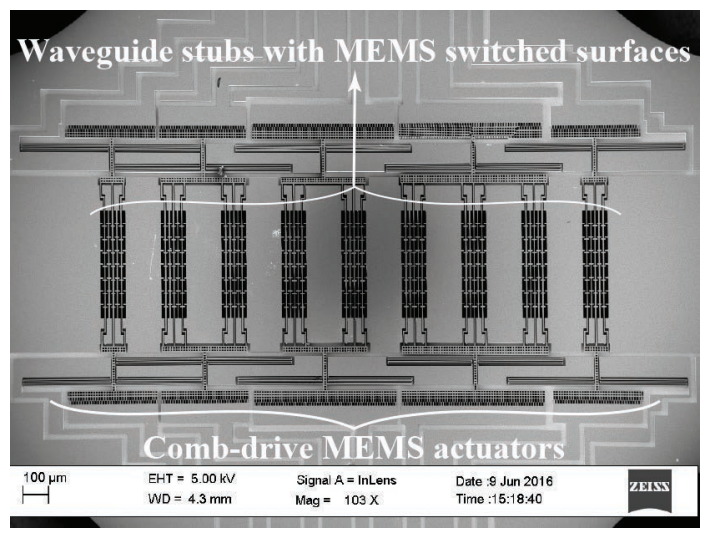

Fig. 8. SEM image of the MEMS switched stub chip with close-up of the MEMS switched surfaces and the comb-drive actuators.

chip size of the MEMS-switched stub chip and the micromachined waveguide chip, including handling areas, contact pads, and bias-lines, is $6.8 \mathrm{~mm} \times 10 \mathrm{~mm}$.

Both the MEMS-switched stub chip and the micromachined waveguide chip have micromachined alignment structures used to align the chips to each other as well as to the machined metal blocks either using micromachined circular or omegashaped alignment structures [18]. Fig. 9 shows the pictures of assembling/mounting the phase shifter. Firstly, the cap chip is placed in the slot on the machined metal block [Fig. 9(a)]. Then the MEMS-switched stub chip is aligned to the machined metal block and placed on top of the cap chip [Fig. 9(b)]. Next the micromachined waveguide chip is aligned and placed on top of the MEMS-switched stub chip [Fig. 9(c)]. Finally, the top metal block is placed and the whole assembly is screwed together [Fig. 9(d)]. Alignment discs are used to align the metal blocks to each other and to the waveguide flanges.
Fig. 9. Assembly/mounting of the phase shifter: (a) cap chip placement; (b) MEMS switched stub chip placement; (c) micromachined waveguide chip placement; and (d) assembled phase shifter.

\section{Characterization AND Analysis}

\section{A. Submillimeter-wave Characterization}

The submillimeter-wave measurements of the fabricated MEMS phase shifter were performed with an Agilent PNAX system using VDI WR-1.5 VNA Extenders calibrated using SOLT calibration. TRL calibration is not used as it is particularly susceptible to alignment errors [19] which reduce the accuracy of the TRL calibration significantly when compared to the fully-flanged standards.

Fig. 10 shows the simulated and measured insertion loss along with the measured return loss for all ten phase states (3.3 bit) of the MEMS-reconfigurable phase shifter. The phase shifter has a broadband frequency response with the insertion loss better than $3 \mathrm{~dB}$ and the return loss better than $15 \mathrm{~dB}$ in the design frequency band of 500-550 GHz. The insertion loss variation of the different phase shifter states is smaller than $0.5 \mathrm{~dB}$. The major part of the insertion loss is caused by the length of the micromachined waveguide and by the interfaces to the flanges. This is shown by the reference measurement of a straight micromachined waveguide without the MEMS switched stubs, included in Fig. 10, showing that the insertion loss attributed by the MEMS-switched surfaces and the stubs is only between 0.5 to $1.5 \mathrm{~dB}$. The measured insertion loss is higher than the simulations which is assumed to be attributed uncertainties in particular in connection with the H-plane split waveguide configuration [20]. The effect of assembly and alignment uncertainties is shown in Fig. 11, displaying the measured insertion loss of 10 different phase shifter chips with fixed phase states, having a reproducibility error of between 2 and $6 \mathrm{~dB}$ instead of $0.5 \mathrm{~dB}$ as anticipated from the measured programmable phase shifter shown in Fig. 10. As verified by simulations shown in Fig. 12, even very small gaps in the chip assembly result in a significant increase of the insertion loss. Furthermore, micromachined static alignment discs were used to perfect surface roughness in the model, and to assembly 


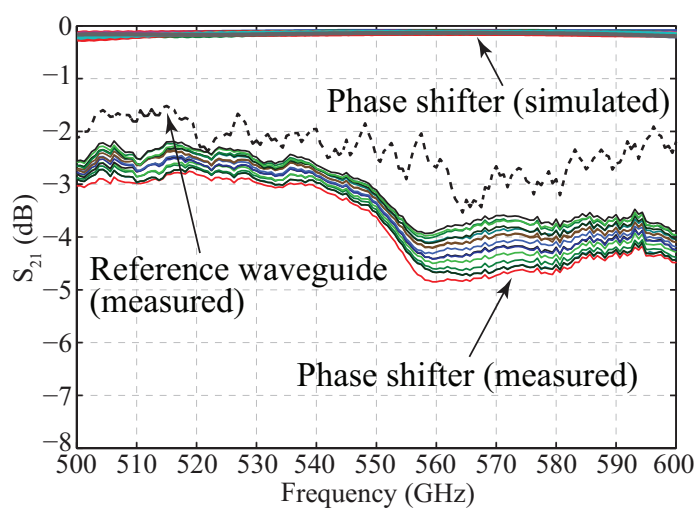

(a)

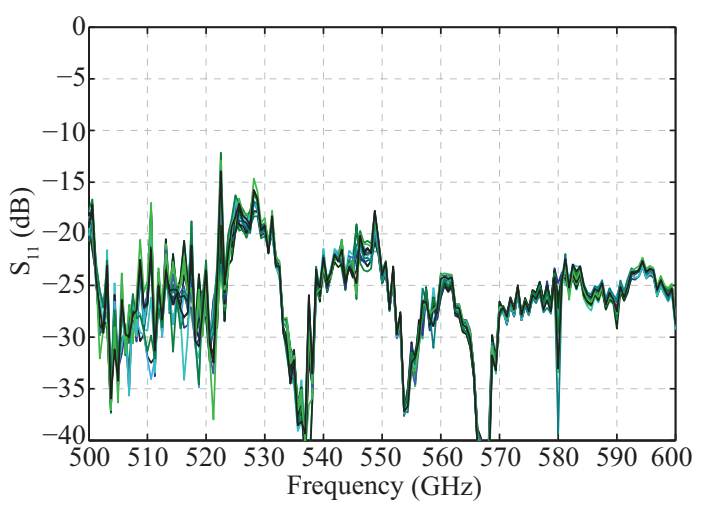

(b)

Fig. 10. Measured S-parameters of the phase shifter for all 10 states (3.3 bit): (a) $S_{21}$; and (b) $S_{11}$. The reference waveguide measurement shows the averaged (linear scale) measurement of four assembly attempts of the same micromachined straight reference waveguide without any MEMS surfaces or stubs.

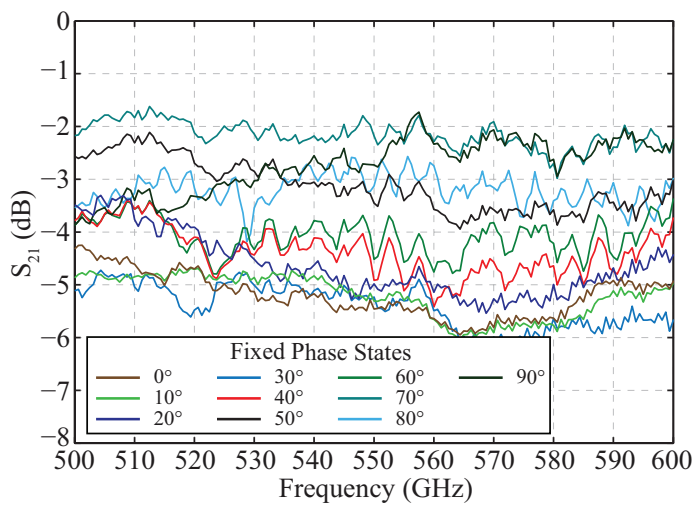

Fig. 11. Assembly alignment uncertainty: Measured insertion loss of 10 chips with different fixed phase states. The variation in the insertion loss is due to the misalignment and assembly uncertainty between the chips and not due to the insertion loss characteristics for different phase states of the phase shifter as shown in Fig. 10.

instead of compression alignment discs [3] for assembling the micromachined chips which results in an alignment error of around $10 \mu \mathrm{m}$, which is, however, according to simulations not significantly contributing to the insertion loss.

The simulated and measured phase shift is normalized to the state with all stubs unblocked and is shown in Fig. 13. The measurements show a linear phase shift of $20^{\circ}$ in ten

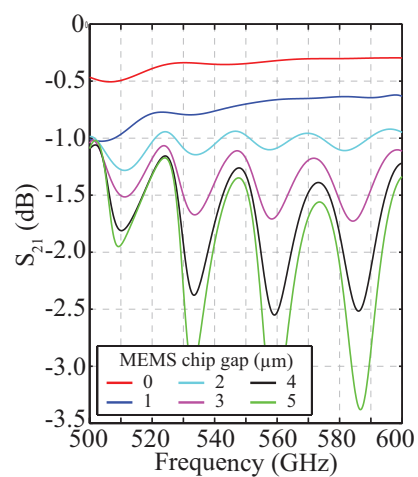

(a)

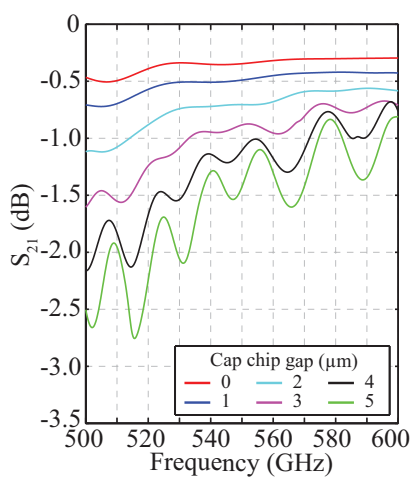

(b)
Fig. 12. Assembly uncertainty: Simulated insertion loss of the phase shifter for different gaps between the chips: (a) gap between the micromachined waveguide chip and the MEMS switched stub chip; and (b) gap between the cap chip and the MEMS switched stub chip.

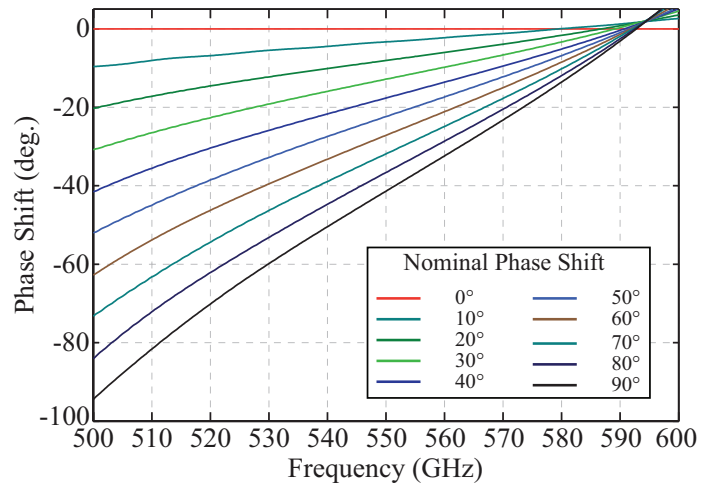

(a)

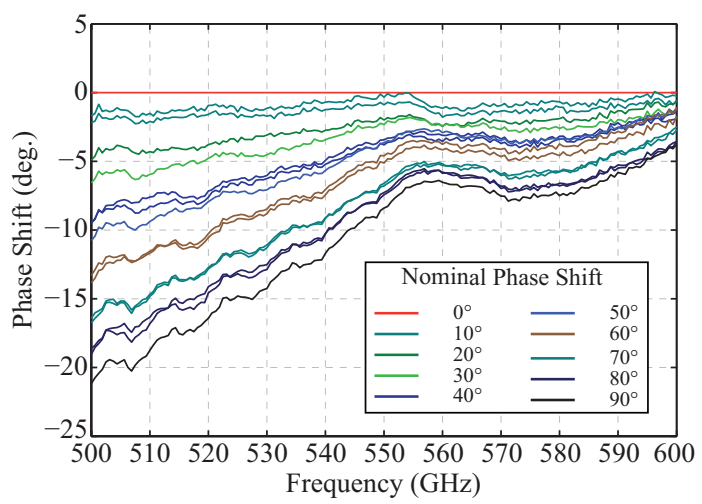

(b)

Fig. 13. Phase shift of the $500-550 \mathrm{GHz} 10$ state (3.3 bit) phase shifter: (a) Nominal phase-shift simulated with CST Microwave Studio; and (b) Measured phase-shift. Multiple lines for same phase shift in (b) demonstrate that the same phase shift can be achieved by different actuator configurations, as explained in section II.

discrete steps (3.3 bit) instead of the designed phase shift of $90^{\circ}$ simulated using CST Microwave Studio. The reason for this discrepancy is the combination of not having an ohmic contact between the vertical cantilevers and the angular sidewall surface profile of the cantilevers due to fabrication inaccuracies. Fig. 3(b) shows that a $20^{\circ}$ phase shift would be achieved for a vertical cantilever gap of $600 \mathrm{~nm}$ when the cantilevers have a straight sidewall surface profile. The same 


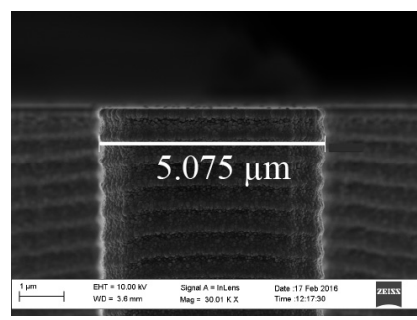

(a)

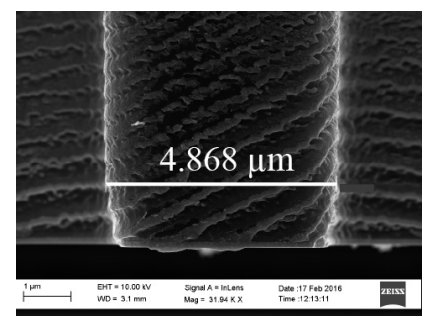

(b)
Fig. 14. Cross-sectional SEM image of the vertical cantilevers, showing a sidewall angle of $0.5^{\circ}$ : (a) top part of the cantilever; and (b) bottom part of the cantilever.

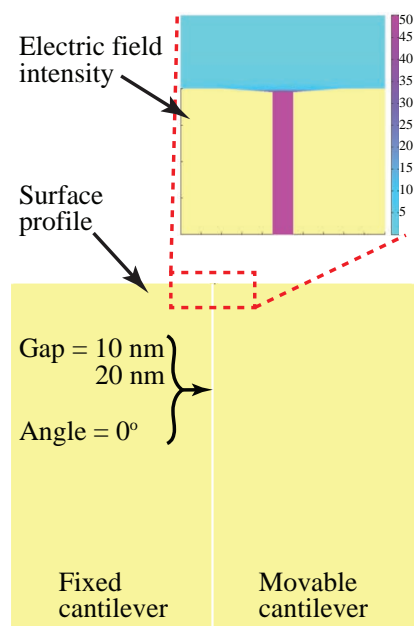

(a)

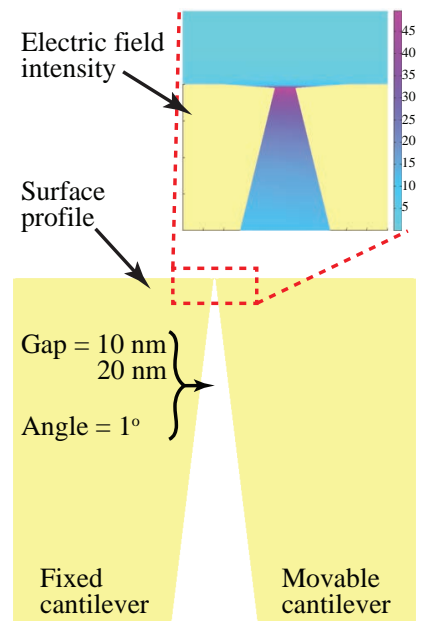

(c)

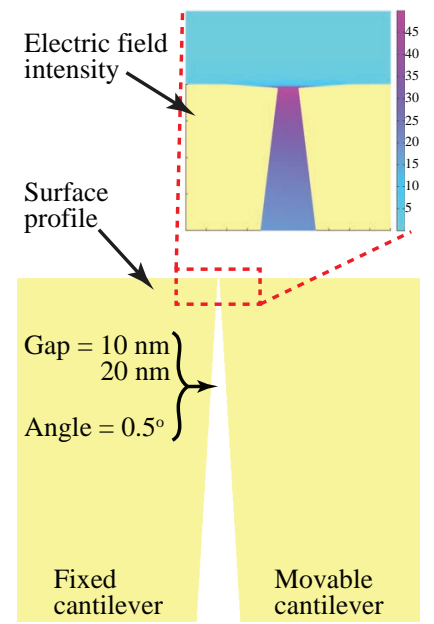

(b)

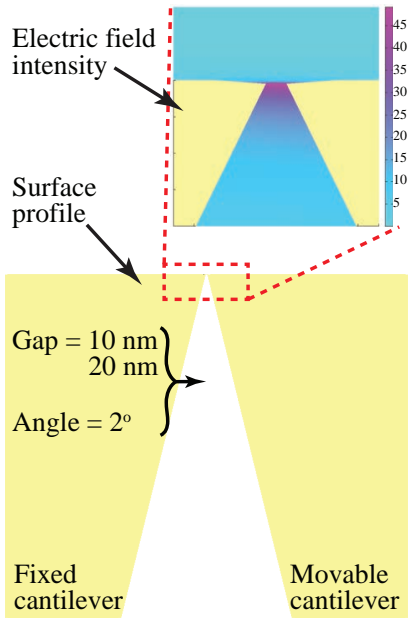

(d)
Fig. 15. Cross-sectional view of the fixed and movable vertical cantilevers used in the phase shift discrepancy analysis: (a) straight surface profile; (b) $0.5^{\circ}$ opening surface profile; (c) $1^{\circ}$ opening surface profile; and (d) $2^{\circ}$ opening surface profile.

phase shift can also be achieved for even smaller gaps due to a slightly angular sidewall profile of the vertical cantilevers. The sidewall angle of the vertical cantilevers is measured to be $0.42^{\circ}$ from the cross-sectional SEM images of the top and bottom part of the vertical cantilevers shown in Fig. 14. The influence of the angular sidewalls of the vertical cantilevers is analyzed by using the Partial Differential Equation (PDE)

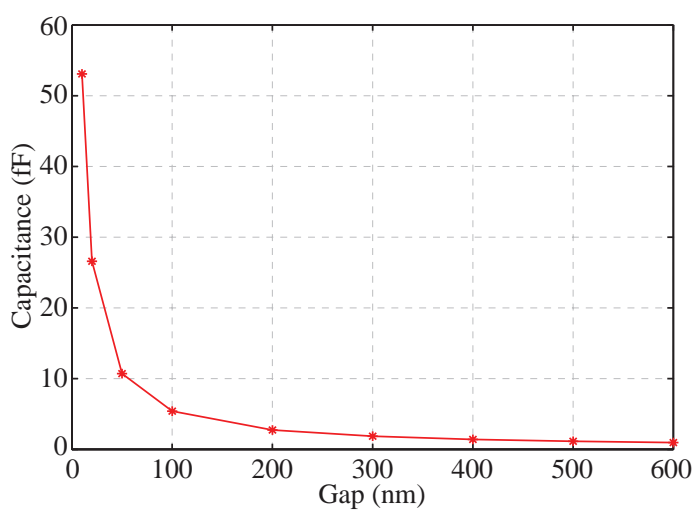

(a)

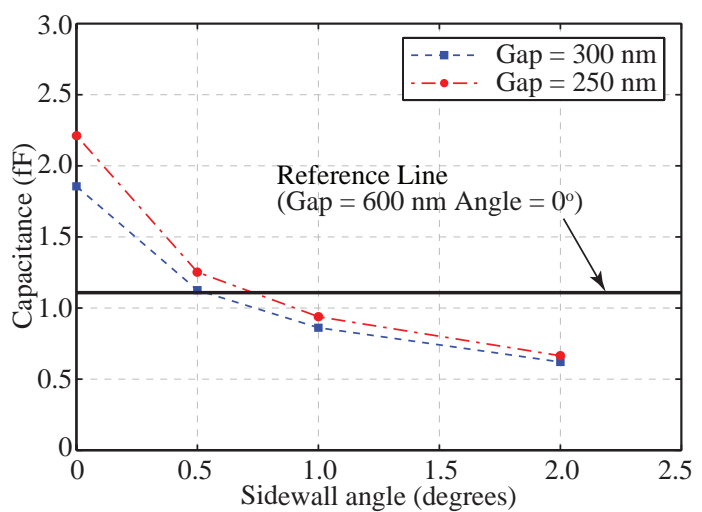

(b)

Fig. 16. Simulated results using Partial Differential Equation (PDE) Toolbox in MATLAB for the electrostatic problem when the vertical cantilevers are close to contact [Fig. 15]: (a) variation in capacitance value vs vertical cantilever gap; and (b) variation in capacitance value vs vertical cantilever sidewall angle. The $600 \mathrm{~nm} / 0^{\circ}$ reference line corresponds to a contact gap which, according to simulations with CST Microwave Studio, results in the same reduced phase shift as measured.

Toolbox in MATLAB and solving the electrostatic problem for the situation when the vertical cantilevers are close to contact. The cross-section of the geometries used for this analysis are shown in Fig. 15 with the inserts showing the electric field intensity at the point where the vertical cantilevers are closest to each other. Fig. 16(a) shows the simulated variation in the capacitance (impedance) value between the vertical cantilevers with a straight sidewall profile with the gap between the cantilevers varying from $10 \mathrm{~nm}$ to $600 \mathrm{~nm}$. Fig. 16(b) shows the variation in the capacitance value for the vertical cantilever gaps of 250 and $300 \mathrm{~nm}$ when the sidewall angle is varied from $0^{\circ}$ to $2^{\circ}$. A capacitance reference line for the vertical cantilever gap of $600 \mathrm{~nm}$ without any sidewall slope (corresponding to the measured reduced phase-shift from $90^{\circ}$ to $20^{\circ}$ ) is also shown. The simulations show that even for a small sidewall angle of $0.5^{\circ}$, the capacitance value for both the 250 and the $300 \mathrm{~nm}$ gaps are very close to the reference line of $600 \mathrm{~nm}$. For $1^{\circ}$ sidewall angle, the capacitance value for both the 250 and the $300 \mathrm{~nm}$ gaps are below the reference line. In conclusion, a sidewall gap of 250 to $300 \mathrm{~nm}$ between the vertical cantilevers due to fabrication inaccuracies is expected to have lowered the phase shift. A gap of a few hundred nanometer can easily be caused by fabrication uncertainty, for instance undercut or 


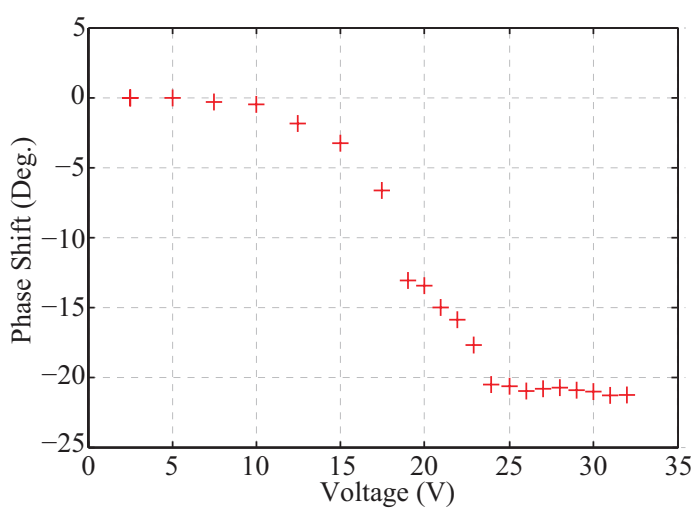

Fig. 17. Measured phase shift vs applied voltage when the phase shifter is operated in the analog mode by ramping the actuation voltage.

large scallops during DRIE, non-optimized lithography step or non-optimized metal step coverage etc., which would result in the vertical cantilevers not making proper ohmic contact. To resolve this issue the overlap of the fixed and movable vertical cantilevers could be increased from the current design of $2 \mu \mathrm{m}$ to at least $6 \mu \mathrm{m}$ or above. This would result in an actual ohmic contact not being needed for achieving the designed phase shift of $90^{\circ}$ at WR-1.5 frequencies. For an overlap of $6 \mu \mathrm{m}$ and higher, it is expected that there is a minimal reduction in the achievable phase shift of $90^{\circ}$ for a $200 \mathrm{~nm}$ gap between the vertical cantilevers when compared to the phase shift reduction observed for a $2 \mu \mathrm{m}$ overlap shown in Fig. 3(b).

The resolution of the phase shifter can also be extended by actuating the MEMS reconfigurable surfaces in an analog mode. This is accomplished by applying simultaneously the same potential on all the comb-drive actuators and measuring the phase at each applied discrete actuation voltage value. Fig. 17 shows the variation in the phase of the phase shifter versus the actuation voltage. The phase shifter design used in these measurements have a measured maximum actuation voltage to contact of $30 \mathrm{~V}$. The maximum phase shift is reached at around $25 \mathrm{~V}$ and further increase of the voltage does not influence the phase shift.

Fig. 18 shows the measured phase shift repeatability for the phase shifter. The phase shifter is utilized in an on/off configuration with either all the stubs blocked (on) or all the stubs unblocked (off). Fig. 18(a) shows good repeatability of the insertion loss during the on/off functioning of the phase shifter over 20 cycles measured in the span of 30 minutes. The measured phase shift during the on/off functioning of the phase shifter over 20 cycles is shown in Fig. 18(b). The drop in the measured phase shift above 15 cycles is assumed to be caused by accidental touching an RF cable, since both the on and the off states have exactly the same phase drop. The phase drift, also affecting both the on and the off states, is caused by the phase drift over time of the VNA and the WR1.5 extenders (specified to be better than $10 \%$ hour).

Fig. 19 shows a cross-sectional image of the stubs and compares the waveguide sidewall roughness at the top and bottom of the stubs. The roughness at the top of the stub is higher than the bottom. This is due to the etching properties of

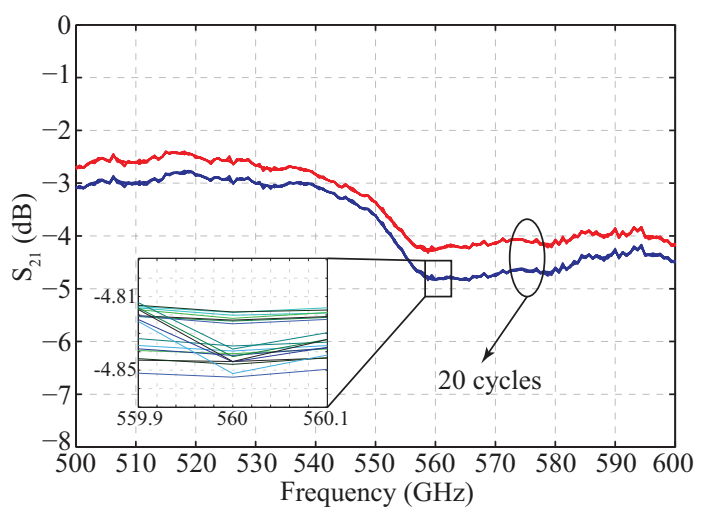

(a)

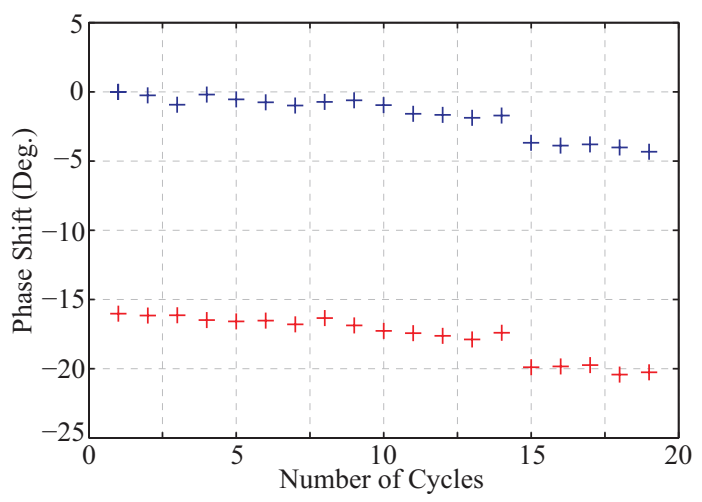

(b)

Fig. 18. Measured repeatability test for the phase shifter: (a) $S_{21}$ repeatability; and (b) phase shift repeatability.

the tool used for DRIE. Fig. 19(b) shows the three-dimensional surface roughness profile of a stub obtained using a Veeco Wyko NT9300 white-light interferometer. The root means square (rms) average roughness, taken over an evaluation area of $15 \times 15 \mu \mathrm{m}$ of a stub shows a roughness of $18.55 \mathrm{~nm}$ at the bottom of the stub and $211.13 \mathrm{~nm}$ at the top of the stub. The higher roughness values could potentially diminish the capability of the stub for creating a phase shift.

\section{B. Electromechanical Characterization and Reliability}

The different comb drive actuator designs are summarized in Table III along with the measured actuation voltages for the five actuators. Actuation voltage repeatability is measured for the Actuator $\mathrm{C}$ of the test Chip 4 [Table III], which has a spring constant of $2.13 \mathrm{~N} / \mathrm{m}$ (average actuation voltage of $21.94 \mathrm{~V}$ ) operated by a comb-drive with 56 fingers without utilizing the push-pull scheme. The measured average actuation voltage, for 20 consecutive cycle measurements, is $21.94 \mathrm{~V}$ with a standard deviation of $0.0503 \mathrm{~V}$.

Lifetime measurements were also performed on Actuator $\mathrm{C}$ of the test Chip 4 [Table III] in an uncontrolled atmospheric environment. The device was cycled with single-side actuation scheme in contact mode with an actuation voltage of $26 \mathrm{~V}$ using a unipolar square waveform with a $50 \%$ duty cycle and a cycle frequency of $100 \mathrm{~Hz}$. The actuation voltage is measured after each decade of actuation cycles and is averaged from three subsequent actuation cycles at each measurement point. 


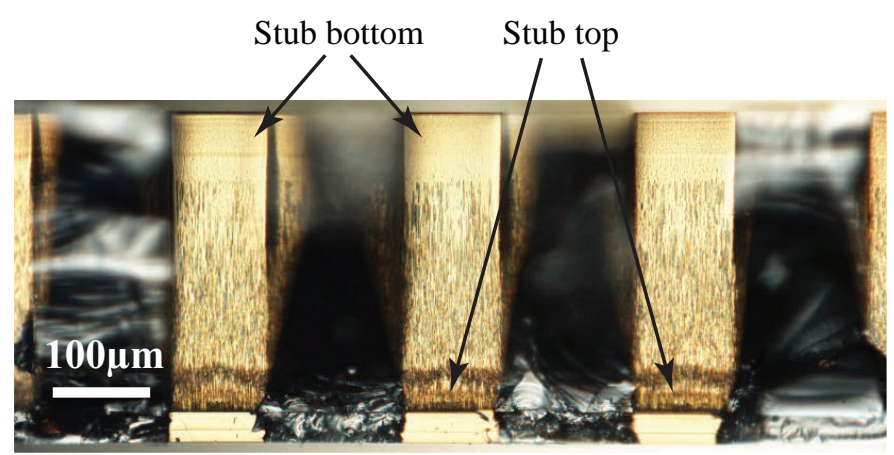

(a)

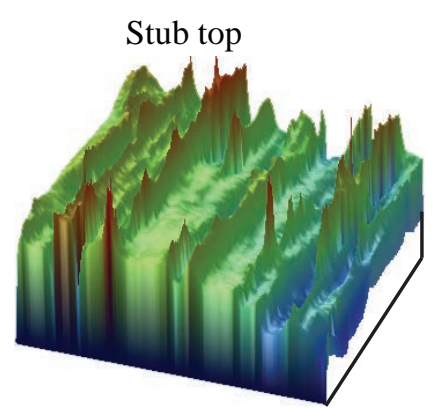

Ra: $168.99 \mathrm{~nm}$

Rq: $211.13 \mathrm{~nm}$

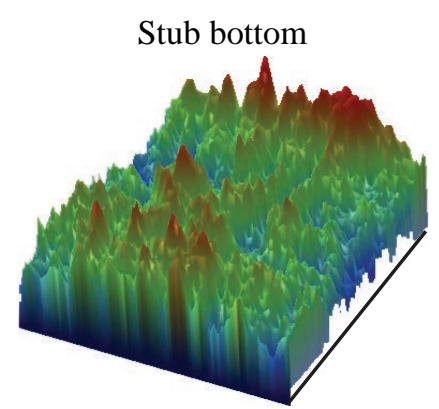

Ra: $14.81 \mathrm{~nm}$ Rq: $18.55 \mathrm{~nm}$ (b)

Fig. 19. Surface roughness profile of the stubs: (a) Cross-sectional image; and (b) roughness data from the white-light interferometer for a sample size of $15 \times 15 \mu \mathrm{m}$.

TABLE III

ACTUATOR DESIGN AND CHARACTERIZATION

\begin{tabular}{|c|c|c|c|c|c|c|c|c|}
\hline \multirow[t]{2}{*}{$\begin{array}{l}\text { Chip } \\
\text { No. }\end{array}$} & \multirow[t]{2}{*}{$\begin{array}{c}\text { Spring } \\
\text { Width } \\
{[\mu \mathrm{m}]}\end{array}$} & \multirow[t]{2}{*}{$\begin{array}{c}\text { Finger } \\
\text { Gap } \\
{[\mu \mathrm{m}]}\end{array}$} & \multirow[t]{2}{*}{$\begin{array}{c}\text { Spring } \\
\text { Constant } \\
{[\mathrm{N} / \mathrm{m}]}\end{array}$} & \multicolumn{5}{|c|}{$\begin{array}{c}\text { Actuation } \\
\text { Voltage } \\
{[\mathrm{V}]}\end{array}$} \\
\hline & & & & $\mathbf{A}$ & B & $\mathbf{C}$ & D & $\mathbf{E}$ \\
\hline 1 & 3 & 2 & 3.67 & 62 & 62 & 50 & 50 & 62 \\
\hline 2 & 2.5 & 2 & 2.13 & 48 & 48 & 38 & 38 & 48 \\
\hline 3 & 2.5 & 1.4 & 2.13 & 39 & 39 & 31 & 31 & 39 \\
\hline 4 & 2.5 & 1 & 2.13 & 30 & 30 & 22 & 22 & 30 \\
\hline 5 & 3 & 1.4 & 3.67 & 51 & 51 & 41 & 41 & 51 \\
\hline 6 & 3 & 1 & 3.67 & 32 & 32 & 26 & 26 & 32 \\
\hline 7 & 4 & 1 & 8.71 & 71 & 71 & 60 & 60 & 71 \\
\hline
\end{tabular}

Fig. 20 shows the measured actuation voltage repeatability test. The actuation voltage remains very stable at $21.9 \mathrm{~V}$ with no detectable variation over the one hundred million cycles. The measurements were stopped after 12 days, when one hundred million cycles were reached without observing any failure, fatigue, or degradation in the actuation voltage. The actuator was also left in the actuated state for 24 hours to analyze the effect of having the comb-drive in the actuated state for a long term. No change in the actuation voltage was observed after the test. This high degree of reliability of a MEMS contactmode device comes from its all-metal, monocrystalline-silicon design.

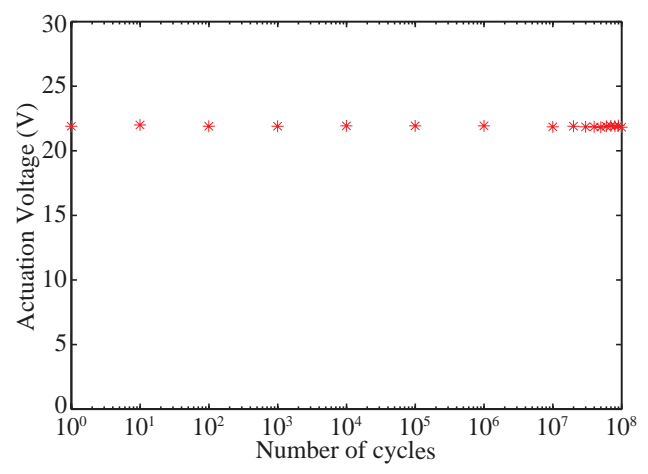

Fig. 20. Life-time characterization: Actuation voltage monitored over 100 million cycles using a $26 \mathrm{~V}$ unipolar square waveform with a $50 \%$ duty cycle and a cycle frequency of $100 \mathrm{~Hz}$ for a device with $\mathrm{k}=2.13 \mathrm{~N} / \mathrm{m}$.

\section{CONCLUSION}

This paper reports the design, fabrication and evaluation of a submillimeter-wave phase shifter in the $500-550 \mathrm{GHz}$ frequency range using MEMS-reconfigurable surfaces. The phase shifter design is based on loading a rectangular waveguide with E-plane stubs and switching the stubs using MEMS reconfigurable surfaces. It consists of three separate micromachined chips aligned and assembled using micromachined alignment structures. A linear phase shift of $20^{\circ}$ has been measured in ten discrete steps. The measured insertion loss is better than $3 \mathrm{~dB}$, of which only 0.5 to $1.5 \mathrm{~dB}$ is attributed to the MEMS switched-surfaces and stubs, and it has been shown that the major part of the loss is caused by assembly and alignment uncertainties. The measured return loss is better than $15 \mathrm{~dB}$. The discrepancy between the measured and simulated phase shift is analyzed in this paper and is attributed to the combination of the angular sidewall surface profile of the vertical cantilevers and not having a proper ohmic contact between the vertical cantilevers. This can be compensated by increasing the overlap between the vertical cantilevers resulting in a approximate capacitive contact being sufficient to achieve the desired phase shift. High actuator reliability with good phase shift repeatability is achieved at medium actuation voltages.

\section{ACKNOWLEDGMENT}

The research described herein is a collaboration effort between KTH Royal Institute of Technology, Stockholm, Sweden, and the Jet Propulsion Laboratory, California Institute of Technology, Pasadena, California, USA, under contract with National Aeronautics and Space Administration.

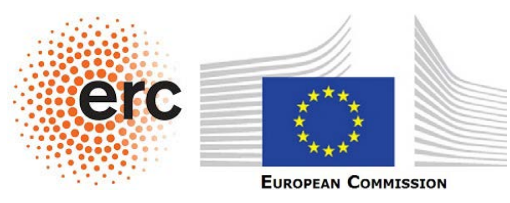

The contribution by $\mathrm{KTH}$ to this work has received funding from the European Research Council (ERC) under the European Union's Horizon 2020 research and innovation programme (grant agreement No 616846), The Swedish Foundation for Strategic Research Synergy Grant Electronics SE13-007, and through a Nils and Hans Backmark scholarship. 


\section{REFERENCES}

[1] P. H. Siegel, "Terahertz technology," IEEE Trans. Microw. Theory Tech., vol. 50, no. 3, pp. 910-928, Mar. 2002.

[2] G. Chattopadhyay, "Technology, capabilities, and performance of low power terahertz sources," IEEE Trans. THz Sci. Technol., vol. 1, no. 1, pp. 33-53, Sep. 2011.

[3] T. J. Reck, C. Jung-Kubiak, J. Gill, and G. Chattopadhyay, "Measurement of silicon micromachined waveguide components at 500-750 GHz," IEEE Trans. THz Sci. Technol., vol. 4, no. 1, pp. 33-38, Jan. 2014.

[4] T. Bryllert, K. B. Cooper, R. J. Dengler, N. Llombart, G. Chattopadhyay, E. Schlecht, J. Gill, C. Lee, A. Skalare, I. Mehdi, and P. H. Siegel, "A $600 \mathrm{GHz}$ imaging radar for concealed objects detection," in IEEE Radar Conf., May 2009, pp. 1-3.

[5] C. A. Leal-Sevillano, T. J. Reck, C. Jung-Kubiak, G. Chattopadhyay, J. A. Ruiz-Cruz, J. R. Montejo-Garai, and J. M. Rebollar, "Silicon micromachined canonical E-plane and H-plane bandpass filters at the terahertz band," IEEE Microw. Wireless Compon. Lett., vol. 23, no. 6, pp. 288-290, Jun. 2013

[6] V. M. Lubecke, K. Mizuno, and G. M. Rebeiz, "Micromachining for terahertz applications," IEEE Trans. Microw. Theory Tech., vol. 46, no. 11, pp. 1821-1831, Nov. 1998.

[7] F. Boussaha, J. Kawamura, J. Stern, and C. Jung-Kubiak, "2.7 THz balanced waveguide HEB mixer," IEEE Trans. THz Sci. Technol., vol. 4, no. 5, pp. 545-551, Sep. 2014

[8] J. Dittloff, F. Arndt, and D. Grauerholz, "Optimum design of waveguide E-plane stub-loaded phase shifters," IEEE Trans. Microw. Theory Tech., vol. 36, no. 3, pp. 582-587, Mar. 1988.

[9] P. Krejcik, "Waveguide stub-line tuning of RF cavities with heavy beam loading," in Proc. Of Particle Accelerator Conf., May 1997, pp. 30303032 .

[10] P. Lampariello, F. Frezza, H. Shigesawa, M. Tsuji, and A. A. Oliner, "A versatile leaky-wave antenna based on stub-loaded rectangular waveguide: part I-theory," IEEE Trans. Antennas Propag., vol. 46, no. 7, pp. 1032-1041, Jul. 1998.

[11] J. Tao, B. Hu, X. Y. He, and Q. J. Wang, "Tunable subwavelength terahertz plasmonic stub waveguide filters," IEEE Trans. Nanotechnol., vol. 12, no. 6, pp. 1191-1197, Nov. 2013.

[12] N. Somjit, G. Stemme, and J. Oberhammer, "Binary-coded 4.25-bit Wband monocrystallinesilicon MEMS multistage dielectric-block phase shifters," IEEE Trans. Microw. Theory Tech., vol. 57, no. 11, pp. 28342840, Nov. 2009.

[13] M. Jost, C. Weickhmann, S. Strunck, A. Gäbler, C. Fritzsch, O. H. Karabey, and R. Jakoby, "Liquid crystal based low-loss phase shifter for W-band frequencies," Electronics Letters, vol. 49, no. 23, pp. 14601462, Nov. 2013

[14] T. Vähä-Heikkilä and M. Ylönen, "G-band distributed microelectromechanical components based on CMOS compatible fabrication," IEEE Trans. Microw. Theory Tech., vol. 56, no. 3, pp. 720-728, Mar. 2008.

[15] U. Shah, E. Decrossas, C. Jung-Kubiak, T. Reck, G. Chattopadhyay, I. Mehdi, and J. Oberhammer1, "500-600 GHz RF MEMS based tunable stub integrated in micromachined rectangular waveguide," in IEEE MTTS Int. Microw. Symp. Dig., May 2015, pp. 1-4.

[16] - " $500-600 \mathrm{GHz}$ submillimeter-wave 3.3 bit RF MEMS phase shifter integrated in micromachined waveguide," in IEEE MTT-S Int. Microw. Symp. Dig., May 2015, pp. 1-4.

[17] Z. Baghchehsaraei and J. Oberhammer, "Parameter analysis of millimeter-wave waveguide switch based on a MEMS reconfigurable surface," IEEE Trans. Microw. Theory Tech., vol. 61, no. 12, pp. 43964404, Dec. 2013.

[18] C. Jung, B. Thomas, C. Lee, A. Peralta, J. Gill, K. Cooper, G. Chattopadhyay, E. Schlecht, R. Lin, and I. Mehdi, "Compact submillimeter-wave receivers made with semiconductor nano-fabrication technologies," in IEEE MTT-S Int. Microw. Symp. Dig., Jun. 2011, pp. 1-4.

[19] D. F. Williams, " $500 \mathrm{GHz}-750 \mathrm{GHz}$ rectangular-waveguide vectornetwork-analyzer calibrations," IEEE Trans. THz Sci. Technol., vol. 1, no. 2, pp. 364-377, Nov. 2011.

[20] J. Hesler, "A photonic crystal joint (PCJ) for metal waveguides," in IEEE MTT-S Int. Microw. Symp. Dig., May 2001, pp. 783-786.

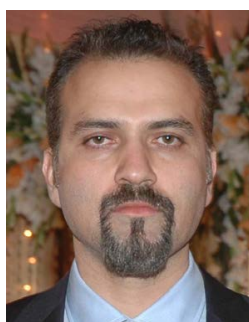

Umer Shah (S'09-M'14) was born in 1981 in Pakistan. He received his BS degree in Engineering from GIK Institute Pakistan in 2003, Master of Science degree in Wireless Engineering from the Technical University of Denmark (DTU) in 2007 and $\mathrm{PhD}$ degree in Microsystem Technology from KTH Royal Institute of Technology, Stockholm, Sweden in 2014. Since May 2014, he is a Postdoctoral researcher in the Micro and Nanosystems group at KTH Royal Institute of Technology. His research focuses includes RF MEMS based filters, phase shifters, matching circuits and antennas.

Umer has authored and co-authored more than 30 reviewed research papers. $\mathrm{He}$ is the recipient of the Best Student Paper Award presented at Asia Pacific Microwave Conference 2010, Yokohama, Japan. Umer is also the recipient of the "2014 IEEE MTT Graduate Fellowship Award" for his research activities.

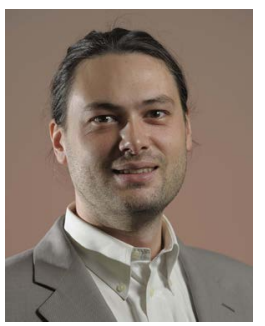

Emmanuel Decrossas (S'08-M'12) received the B.S. and M.S with honors in engineering science and electrical engineering from the universit Pierre et Marie Curie Paris-6, Paris, France in 2004 and 2006 respectively. In 2012, He received his Ph.D. from the University of Arkansas, Fayetteville in electrical engineering.

He authored and co-authored over 30 technical journal articles and conference papers, has written one book chapter, and also holds over 6 patents. Emmanuel Decrossas was awarded the NASA postdoctoral fellowship given annually to only 60 postdoctoral candidates worldwide, based on the scientific merit of proposed research, academic and research record in 2012.

Although he joined JPL only in 2015, he has already delivered a large amount of flight hardware for Mars CubeSat One (MARCO), and flight designs for Sentinel-6 (ex JASON-CS), Cold Atom Laboratory (CAL) and $\mathrm{THz}$ instruments. He is currently developing the high power hat cover for SWOT Karin instrument, the antennas for EUROPA REASON radar instruments, NISAR GPS, DHFR CubeSat and is involved in several R\&D project such as ground penetrating radar, radio occultation antennas for CubeSat and balloon ExaVolt Antenna (EVA).

Dr. Decrossas is member of the electrical engineering honor society Eta Kappa Nu, IEEE and in 2013 he was elected secretary of the IEEE EMC technical committee TC-11 on nanotechnology and advanced material.

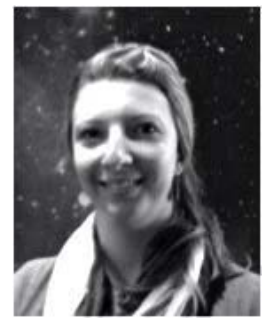

Cecile Jung-Kubiak (M'15) received a masters degree in Physics and Materials Chemistry from PolytechMontpellier, France in 2006 and the $\mathrm{PhD}$ degree in Physics from Universite Paris-Sud XI, France in 2009. She was the recipient of a 2-year NASA Postdoctoral Fellowship at the California Institute of Technology in 2010, and she is now a member of the Technical Staff for the S.W.A.T. group of the Jet Propulsion Laboratory, in Pasadena, USA. Her research interests include the development of silicon micromachining technologies using DRIE techniques, the miniaturization of multi-pixel arrays to build compact 3-D instruments and GaAs-based frequency multipliers and mixers in the $\mathrm{THz}$ region. She has coauthored over 50 papers in international journals and conferences, holds several patents and was a recipient of the 2010 JPL Outstanding Postdoctoral Research Award in the field Technology, Instrumentation, and Engineering and the 2014 IEEE THz Science and Technology Best Paper Award. 


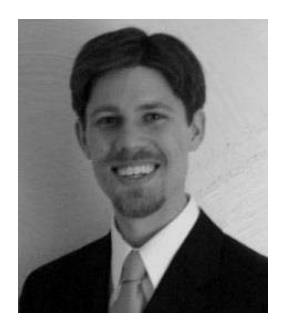

Theodore Reck (M'04-SM'15) received the B.S. degree in electrical engineering from the University of Texas at Austin in 2000 and $\mathrm{PhD}$ in Electrical Engineering at the University of Virginia in 2010. From 2010 to 2013 he was a NASA postdoctoral fellow at the Jet Propulsion Laboratory (JPL) designing terahertz devices that utilize silicon micromachining.

Presently he is a member of the technical staff at JPL. His research interests also include RF-MEMS, antenna arrays, terahertz metrology, and cryogenic MMIC LNAs.

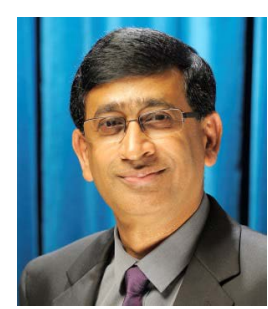

Goutam Chattopadhyay (S'93-M'99-SM'01-F'11) is a Senior Research Scientist at the NASAs Jet Propulsion Laboratory, California Institute of Technology, and a Visiting Associate at the Division of Physics, Mathematics, and Astronomy at the California Institute of Technology, Pasadena, USA. He received the B.E. degree in electronics and telecommunication engineering from the Bengal Engineering College, Calcutta University, Calcutta, India, in 1987, the M.S. degree in electrical engineering from the University of Virginia, Charlottesville, in 1994, and the Ph.D. degree in electrical engineering from the California Institute of Technology (Caltech), Pasadena, in 1999. From 1987 until 1992, he was a Design Engineer with the Tata Institute of Fundamental Research (TIFR), Pune, India.

His research interests include microwave, millimeter-, and submillimeterwave heterodyne and direct detector receivers, frequency sources and mixers in the terahertz region, antennas, SIS mixer technology, direct detector bolometer instruments; InP HEMT amplifiers, mixers, and multipliers; high frequency radars, and applications of nanotechnology at terahertz frequencies. He has more than 250 publications in international journals and conferences and holds more than fifteen patents. Among various awards and honors, he was the recipient of the Best Undergraduate Student Award from the University of Calcutta in 1987, the Jawaharlal Nehru Fellowship Award from the Government of India in 1992, and the IEEE MTT-S Graduate Fellowship Award in 1997. He was the recipient of the best journal paper award in 2013 by IEEE Transactions on Terahertz Science and Technology, and IETE Prof. S. N. Mitra Memorial Award in 2014. He also received more than 30 NASA technical achievement and new technology invention awards. He is an associate editor of the IEEE Transactions on Antennas and Propagation, a Fellow of IEEE (USA) and IETE (India) and an IEEE Distinguished Lecturer.

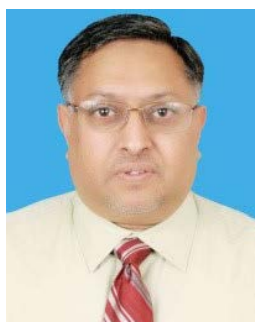

Imran Mehdi (S'83-M'90-SM'05-F'10) received the B.S.E.E., M.S.E.E., and Ph.D. degrees from the University of Michigan, MI, USA, in 1985, 1986, and 1990, respectively.

He is a Senior Research Scientist at the Jet Propulsion Laboratory, California Institute of Technology. He joined JPL in 1990 and is currently a Group Supervisor in the Instrument Electronics and Sensors Section. His responsibilities include developing $\mathrm{THz}$ components, technologies and subsystems for current and future NASA missions. These devices and components were implemented on the ozone monitoring Microwave Limb Sounder (MLS) instrument which is still operational as well as the (Microwave Instrument on Rosetta Orbiter) MIRO instrument which represents the first submillimeter-wave receiver operational in deep space. From 1999 he led the effort of developing broadband solid-state sources from 200 to 2500 $\mathrm{GHz}$ for the Heterodyne Instrument for Far Infrared (HIFI) on the Herschel Space Observatory, a cornerstone European Space Agency mission. HIFI has successfully completed its mission enabling ground breaking astrophysics observations. He is an IEEE Fellow and serves as a topical editor for the IEEE Transactions on $\mathrm{THz}$ Science and Technology. His current interests include millimeter and submillimeter-wave devices and technology, nanotechnology, high-frequency instrumentation, 3-D Submm-wave systems, and development of compact, low-power heterodyne receivers for planetary missions.

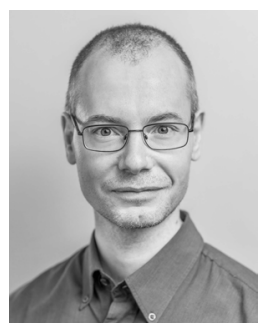

Joachim Oberhammer (M'06-SM'12), born in Italy in 1976; M.Sc. EE from Graz University of Technology, Austria, in 2000; Ph.D. from KTH Royal Institute of Technology in Stockholm, Sweden, in 2004. Post-doctoral research fellow at Nanyang Technological University, Singapore, in 2004, and at Kyoto University, Japan, in 2008. Since 2005 leading radio-frequency/microwave/terahertz micro-electromechanical systems research at $\mathrm{KTH}$; Associate Professor at KTH in 2010; Professor in Microwave and THz Microsystems at KTH since 2015. Guest researcher at Nanyang Technological University, Singapore, in 2007; guest researcher at NASA-Jet Propulsion Laboratory, USA, in 2014.

$\mathrm{He}$ is author and co-author of more than 100 reviewed research papers and holds 4 patents. In 2004, 2007, and 2008 he got an award by the Ericsson Research Foundation, a grant by the Swedish Innovation Bridge, and a scholarship by the Japanese Society for the Promotion of Science, respectively. The research work he is heading received six Best Paper Awards (five of which at IEEE conferences), and four IEEE Graduate Fellowship Awards (by MTT-S and by AP-S) since 2009. He served as TPRC member of IEEE Transducers 2009 and 2015, IEEE International Microwave Symposiums 2010-2016, IEEE Micro Electro Mechanical Systems 2011 and 2012, and IEEE Radio and Wireless Week 2015 and 2016. Dr Oberhammer is Steering Group member of the IEEE MTT-S and AP-S Chapers Sweden since 2009. In 2013, he received an ERC Consolidator Grant by the European Research Council. Since 2014 he is Steering Group Member of the Young Academy of Sweden. 